\title{
Orbital and intracranial complications after acute rhinosinusitis*
}

\author{
Jan Kastner ${ }^{1}$, Milos Taudy ${ }^{1}$, Jiri Lisy ${ }^{2}$, Paul Grabec ${ }^{3}$, Jan Betka ${ }^{1}$ \\ 1 Department of Otorhinolaryngology and Head and Neck Surgery, University Hospital Motol, 1st Medical \\ Faculty, Charles University, Prague, Czech Republic \\ 2 Department of Imaging Methods, University Hospital Motol , 2nd Medical Faculty, Charles University, \\ Prague, Czech Republic \\ 3 Department of Otorhinolaryngology, General Hospital, 1st Medical Faculty, Charles University, Prague, \\ Czech Republic
}

\begin{abstract}
SUMMARY Background/objectives: Nowadays, intracranial abscess is a rare complication of acute rhinosinusitis. The consequent orbital and intracranial complications of acute rhinosinusitis are rare but must be mutually excluded in complicated rhinosinusitis even when proper surgical and medical treatment tend to efficiently heal the orbital complication.

Methods: We report a case of a patient who primarily revealed symptoms of orbitocellulitis as a complication of odontogenous rhinosinusitis. Proper diagnostic and therapeutical measures were undertaken to manage the disease immediately after stationary admission. We strongly rely on a literature review and recommendations on diagnosis and treatment of this possibly lethal complication in the discussion.

Results: Two weeks after an inconspicuous healing period, hemiparesis due to formation of an intracranial abscess developed. An emergency situation revealed the unusual character of the clinical situation. In a literature review concerning intracranial complication after acute rhinosinusitis, we demonstrate the complexity of this complication. We indicate the predisposing factors, diagnostic measures and treatment strategy.

Conclusion: The possible role of underlying mechanisms of intracranial abscess formation is discussed. The correct indication of imaging methods and accurate evaluation of diminutive symptoms are essential. We recommend performing a complementary CT of the brain or MRI, even when a previous CT scan of the orbit/paranasal sinuses reveals no cerebral pathology, to avoid or minimize the number of future patients with consecutive orbital and intracranial complications of acute rhinosinusitis.
\end{abstract}

Key words: acute rhinosinusitis, intracranial complication, orbitocellulitis, endoscopic drainage, odontogenous

\section{INTRODUCTION}

Rhinosinusitis is clinically defined as inflammation of the nose and paranasal sinuses characterised by two or more symptoms, one of which should be either nasal obstruction or nasal discharge, along with facial pressure and/or loss of smell with positive endoscopic findings and/or CT pathology. In acute rhinosinusitis, the symptoms last less than 12 weeks; clinical ENT examination involves nasal endoscopy. CT scan is not recommended unless the course of disease is very severe, if the patient is immunocompromised, or signs of complications are present ${ }^{(1)}$.

Complications of rhinosinusitis might be divided into orbital, osseous and intracranial. Orbital involvement is divided according to the Chandler classification into: preseptal inflam- matory oedema (periorbital cellulitis), postseptal orbital cellulitis, subperiosteal abscess, orbital abscess, and other complications (cavernous sinus thrombosis, etc.). Intracranial complications include either phlegmonous inflammation (meningitis, cerebritis) or abscess formation (epidural, subdural, intracerebral), and cavernous sinus thrombosis. Osseous complications are represented by frontal bone osteomyelitis. Their incidence has dramatically decreased when compared to the preantiobiotic era, but it is estimated that in patients affected by acute bacterial rhinosinusitis with intracranial spread, in spite of antibiotic therapy, there is still a high incidence of morbidity, and the mortality rate is between $5 \%$ and $10 \%{ }^{(2)}$. The spread of infection into the orbit and intracranially follows natural paths or predisposed routes, (i.e. pathogens can pass through the veins or alternatively, they may extend behind sinuses directly 
through a dehiscence (e.g. in the lamina papyracea) or by eroding the sinus bones) ${ }^{(3,4)}$.

Diagnostic imaging should be done urgently, under hospitalization and it should rely on clinical and laboratory (white blood count) examinations, nasal endoscopy, and especially CT scan. Symptomatology of rhinosinusitis may vary, but whenever there is periorbital oedema, a displaced bulb, double- or reduced-vision, ophthalmoplegia or severe frontal headache, frontal swelling, signs of meningitis, focal neurological signs or systemic signs, one must be aware of these complications of rhinosinusitis and perform urgent investigations and intervention. These include a good interdisciplinary co-operation between otorhinolaryngology, ophthalmology, neurology, radiology, and possibly even maxillofacial surgery and paediatrics.

The essential treatment of orbital cellulitis, which means a phlegmonous infection posterior to the orbital septum without abscess formation (stage II, according to the Chandler classification), is surgical drainage of the predisposing rhinosinusitis and concurrent parenteral antibiotics. The preferred surgical drainage nowadays is via an endoscopic approach in both children and in adults ${ }^{(5)}$.

\section{CASE REPORT}

A female patient, 30 years old, was sent to the ENT Department of Motol Teaching Hospital, 1st Medical Faculty of Charles University in Prague, from the Ophthalmology Department with suspected unilateral orbitocellulitis. There was an obvious unilateral lid oedema on her left side, slight proptosis and ophthalmoplegia with diplopia, but no visual field loss or vision deterioration. She did not suffer from nasal obstruction, but only minimal nasal discharge and orbital and facial pressure on her left side. The duration of symptoms and ocular signs formation developed over two days. The patient belonged to an average socioeconomic status, with no previous comorbidity, and was not immunocompromised. Nasal endoscopy revealed a yellowish mucus in the middle meatus and mucosal swelling on the left side with no foetor. She was admitted, and blood analysis and CT scans were performed (Figure 1). The patient was subfebrile, with no chills. The white blood count revealed leukocytosis (22 000/L) and an

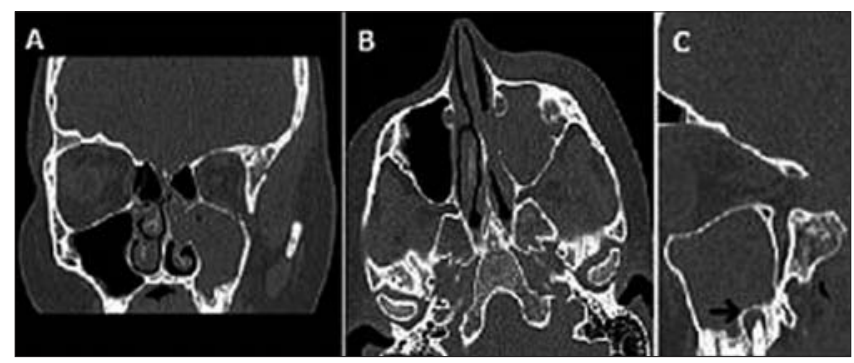

Figure 1. Coronary (A), axial (B) and sagittal (C) projections in a patient with left-sided odontogenous (black arrow) unilateral acute rhinosinusitis complicated by orbitocellulitis (day 0 - preoperatively).

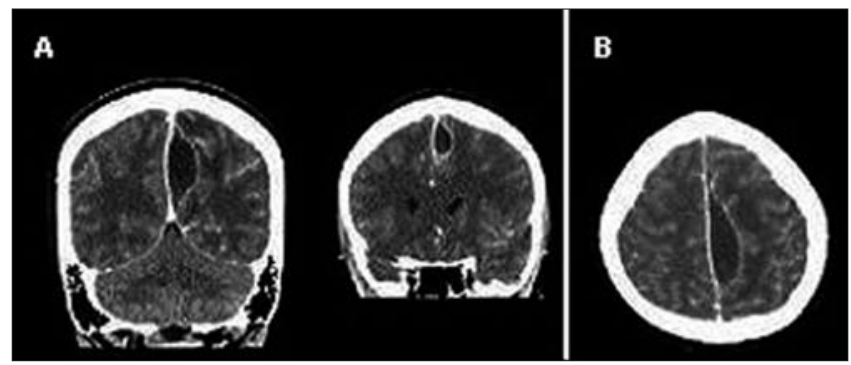

Figure 2. Intracranial abscess formation in various planes of coronary (A) and axial (B) projections (day 20 postoperatively).

extremely high $\mathrm{C}$-reactive protein $(\mathrm{CRP}=315)$. Parenteral antiobiotics (cefuroxim axetil $750 \mathrm{mg}$ every 8 hours) were administered immediately. CT scans (paranasal sinuses projections: coronary, axial, sagittal) showed a maxillary sinusitis on the left side with the $1^{\text {st }}$ and $2^{\text {nd }}$ molar roots protruding into the maxillary sinus with no intraorbital abscess formation, just an effusion of the pre- and postseptal orbital soft tissue components). Stomatology examination was performed; it revealed a presumed necrotic $1^{\text {st }}$ molar. On the very day of admission, surgery under general anaesthesia was performed in terms of endoscopic sinus surgery on the left side (partial anterior ethmoidectomy and supraturbinal antrostomy) and necrotic molar extraction. Swabs (inclusive for anaerobic infection) of the maxillary sinus empyema was taken and according to probable odontegenous etiology, parenteral antibiotics were changed from cefuroxim to a combination of clindamycin $(600 \mathrm{mg}$ every 8 hours) and gentamycin $(240 \mathrm{mg} /$ day for 5 days $)$. However, the swab revealed no positive results after two days of cultivation, therefore the antibiotics combination was consulted with the microbiology center of our hospital.

The postoperative healing period was unconspicuous, free of any evident complication. The patient was not febrile or subfebrile from the $1^{\text {st }}$ postoperative day, the periocular oedema was in regression, and there was only residual crusting in the left nasal cavity with no putrid secretion. The only one residual symptom remaining was a slight pressure located in her left eye. A neurology and ophthalmology check-up were administered, but they revealed no pathology ("fully regular findings") as well as stomatology check-up. The blood test before discharge displayed normal or almost normal findings (leuk. $7000 / \mathrm{L}, \mathrm{CRP}$ 14) and the patient was released on the $7^{\text {th }}$ postoperative day, still continuing with peroral antibiotics intake (clindamycin 300mg every 8 hours) for another week. The patient was seen twice more (day 10 and 15 postoperatively) at the out-patient unit for cleaning her left nasal cavity of residual crusting (no nasal discharge or symptoms re-manifestation was recorded) and to review the definite histology (benign nasal/paranasal mucosa).

On day 20 postoperatively, she was re-admitted urgently because a hemiparesis on the right side developed. Brain CT 

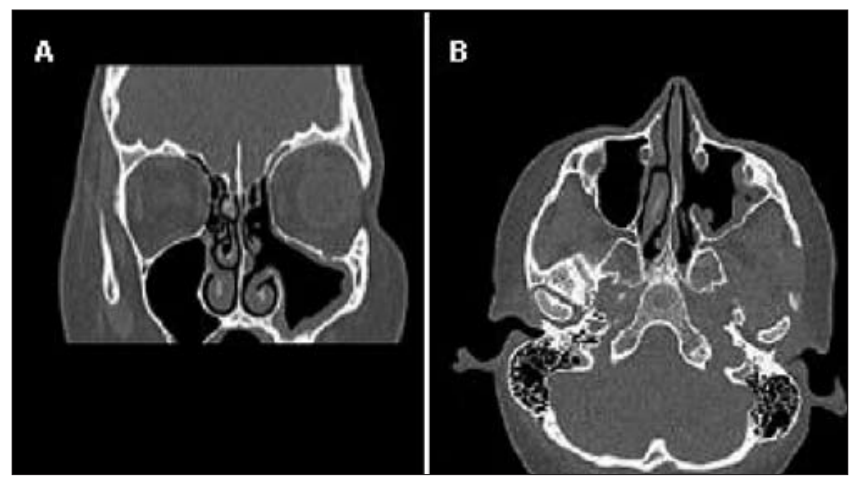

Figure 3. Coronary (A) and axial (B) projections documenting the post endoscopic sinus surgery result (day 21 postoperatively).

revealed signs of meningitis (loss of subarachnoidal space and contrast saturation of pial veins) and an intracranial (subdural) abscess formation in the parafalcinic area left, i.e. interhemispheric empyema (Figure 2). Postoperative CT scans (taken on day 21 postoperatively) showed a good result of sinus surgery (Figure 3).

Proper neurology, neurosurgery (craniotomy for subdural abscess) and medical measures were undertaken to manage the intracranial complication. The abscess fluid cultivation (for aerobes, anaerobes, fungi) was negative again. Blood culture was negative. No immunodeficiency was found. The patient still suffered from minimal residual neurological sequelae (residual motoric lesion on the right side) three months after hemiparesis manifestation.

\section{DISCUSSION}

Coincidence of orbital and intracranial complication of acute rhinisinusitis has been described very marginally in the literature. Most studies encompass orbital or intracranial complication separately, many include acute and chronic rhinosinusitis together, and the majority focuses on children.

An interesting distribution of rhinosinusitis complications is seen upon demographic assessment. Two famous NorthAmerican studies on intracranial complications in rhinosinusitis in a predominantly adult, and young adult population and children do not handle orbital co-complications separately ${ }^{(4,6)}$. Only 2 out of 24 patients (8.3\%) in Clayman's study suffered from subsequent symptoms associated with intracranial complications typical for orbitocellulitis (ophthalmoplegia and decreased visual acuity) as well ${ }^{(4)}$. Another retrospective study that focused on intracranial complications of rhinosinisutis revealed that in 3 of 15 patients (20\%) there was additional subperiosteal abscess formation in the orbit ${ }^{(7)}$. One of the latest North-American studies documented only about 4 cases of intracranial complications among 74 paediatric patients who were admitted for orbital infection $(5.4 \%){ }^{(8)}$. Only 1 of 52 patients $(1,9 \%)$ with orbital cellulitis/orbital abscess subsequently developed an intracranial complication in a large retro- spective study from Australia (meningitis) ${ }^{(9)}$. Some studies from Africa have detailed a relatively large number of complications in rhinosinusitis, probably due to the lower availability of antibiotics administered for acute rhinosinusitis. One study from the region of West Africa revealed that orbitocellulitis developed in 47 of $90(52 \%)$ patients admitted for rhinosinusitis. In 10 of 47 (19\%) suffering from orbitocellulitis, a subsequent intracranial complication developed (cavernous sinus thrombosis, meningitis, etc.) ${ }^{(10)}$. Another study from South Africa has reported the distribution of complications in 59 patients (children and young adults) with complicated rhinosinusitis: intracranial: 36 patients $(61 \%)$, orbital: 13 (22\%) and both intra-cranial and orbital: 10 patients (17\%). There was a mortality of $5 \%$ (3 of 59 patients) ${ }^{(1)}$. A large retrospective study concerning rhinogenic intracranial complications has shown that despite advances of modern medicine, a relatively high mortality of $16 \%$ is present ( 35 from 219 patients) with a prevalence of meningitis followed by brain abscess and subdural empyema ${ }^{(12)}$. In a study from the Middle East, 9 of 116 patients $(8 \%)$ developed an orbital abscess (after predisposing rhinisinusitis and trauma) and an intracranial complication ${ }^{(13)}$. A study from Israel on orbital complications secondary to acute rhinosinusitis in children aged 2 and younger strongly relied on a meticulous multidisciplinary follow-up in a hospital. Surgical intervention was avoided in the majority of cases with proper diagnosis (only 1 from 52 was operated) ${ }^{(14)}$. In Europe, 25 patients with complicated rhinosinusitis were followed up in a German study. The percentage of complications showed $20(80 \%)$ patients with orbital complications, $3(12 \%)$ with intracranial complications, and $2(8 \%)$ with both orbital and intracranial complications ${ }^{(15)}$.

Consequently, no matter in what region of the world, there exists a group of patients with co-existing orbital and intracranial complications in rhinosinusitis, or where orbital complications of rhinosinusitis account for predisposing factors for developing intracranial complications ${ }^{(16,17)}$. The neurological symptomatology is often mild or absent. Only one paper has stressed the role of MRI in early detection of intracranial cocomplications ${ }^{(8)}$. All other studies strongly suggested relying on CT scanning, while the study by Herrmann ${ }^{(8)}$ emphasized adjuvant MRI scanning due to some false negative results of CT scans in patients with subsequent intracranial abscess. MRI is advisable in patients with positive symptomatology ("changes in mentation", persistent fever) or indefinite CT findings. Some guidelines for the management of periorbital cellulitis/abscess have been proposed in Europe in the past ${ }^{(18,19)}$. Standard CT scans done immediately for all cases of complicated rhinosinusitis after admission was not fully stressed. But EP ${ }^{3}$ OS 2007 (= European position paper on rhinosinusitis and nasal polyps published by the European Rhinologic Society in 2007) has established that CT scanning is the preferred method of imaging in rhinosinusitis ${ }^{(1)}$. CT scans in adults as well as in children upon admission is considered as "conditio sine qua non". 
When one examines the results of studies on complications of rhinosinusitis, it is evident that orbital complications exceed intracranial complications by up to $20 \%$. We propose performing an additional CT or MRI of the brain in patients admitted with orbital complications of rhinosinusitis despite negative brain pathology on the initial CT of the orbit/paranasal sinuses. Due to the different settings of CT of the orbit and CT of the brain, it was impossible to detect interhemispheric subdural empyema in an atypical location in our patient.

The usual microbiological profile seems to be the same in all continents, with a prevalence of Streptococcus, Staphylococcus, anaerobes (e.g. Fusobacterium) present. Fungi is prevalent in immunocompromised individuals. A blood culture seems to be ineffective in the majority of cases. There might be a large number of negative cultures due to antibiotic pretreatment or cultivation failure. Possible future trends in revealing infectious agents are implemented in terms of molecular genetic methods ${ }^{(20)}$.

The predominance of complications in patients during the second and third decades of life is thought to be associated with a peak in the vascularity of the bony diploic system (and thus continued growth of the frontal sinus). Understandably, this implies that retrograde septic thrombophlebitis is the most likely route of infection. The direct or hematogenous routes are less common; atypical locations of the intracranial abscess might be found with hematogenous spread. The predominant location of complicated rhinosinusitis is the frontoethmoidal region. The maxillary sinus is predominantly affected in odontogenous rhinosinusitis; conversely, pansinusitis is surprisingly seldomly seen.

Management of individual complications has been well established. These consist of medical measures (i.e. stationary treatment, parenteral broad spectrum antibiotics (for anaerobes as well) and surgical drainage of abscesses. Endoscopic sinus surgery is preferred over the external approach in surgical drainage of predisposing rhinosinusitis. A multidisciplinary approach is required in orbital or intracranial abscess formation. Standard treatment does not involve corticosteroids: these are administered only if the patient may benefit from reducing intracranial or intraorbital oedema ${ }^{(21)}$.

Intracranial infections still account for serious morbidity and mortality rates. In addition, the postoperative sequelae with residual neurological symptoms often imply disaster for a previously healthy, mostly young, adult population.

\section{CONCLUSION}

The diagnostic and therapeutic management of orbital complications of rhinosinusitis has been fully established. Nevetheless, some patients, especially young adults, suffer from subsequent intracranial complications. A co-existing/con- sequent intracranial complication affects this group of patients with orbital complications of rhinosinusitis in up $20 \%$ of cases according to reviews of the scientific literature. That is probably due to the aggressiveness of the infection or spread via anatomical channels. Proper treatment measures may mask these predisposing factors, and therefore in general, if one of the complications appears, the others must be excluded.

We recommend to conclude treatment by performing a CT of the brain or MRI when an orbital complication has occurred following rhinosinusitis despite a negative brain pathology in CT scans of the orbit and paranasal sinuses and/or minimal or absent symptomatology before dismission from inhouse treatment.

\section{ACKNOWLEDGEMENTS}

To Professor Daniel Simmen (ORL-Zentrum, Klinik Hirslanden, Zurich, Switzerland) and to Professor Eduard Zverina (Neurosurgical unit, Dept. of Otorhinolaryngology and Head and Neck Surgery, University Hospital Motol, $1^{\text {st }}$ Medical Faculty, Charles University, Prague, Czech Republic) for their help, lectures and valuable consultations.

Supported by grant GAUK 203435 36/06 and IGA 9908-3 (Czech Republic).

\section{REFERENCES}

1. Fokkens WJ, Lund VJ, Mullol J, et al. European Position Paper on Rhinosinusitis and Nasal Polyps 2007. Rhinology 2007; 45 suppl 20: $131-136$.

2. Chandler JR, Langenbrunner DJ, Stevens ER. The pathogenesis of orbital complications in acute sinusitis. Laryngoscope 1970; 80: 1414-1428.

3. Lang EE, Curran AJ, Patil N, Walsh RM, Rawluk D, Walsh MA. Intracranial complications of acute frontal sinusitis. Clin Otolaryngol 2001; 26: 452-457.

4. Clayman GL, Adams GL, Paugh DR, Koopmann CF, Jr. Intracranial complications of paranasal sinusitis: a combined institutional review. Laryngoscope 1991; 101: 234-239.

5. Mortimore S, Wormald PJ. Management of acute complicated sinusitis: a 5-year review. Otolaryngol Head Neck Surg 1999; 121: 639-642.

6. Lerner DN, Choi SS, Zalzal GH, Johnson DL.Intracranial complications of sinusitis in childhood.Ann Otol Rhinol Laryngol 1995; 104: 288-293

7. Gallagher RM, Gross CW, Phillips, CD. Suppurative Intracranial Complications of Sinusitis Laryngoscope 1998; 108: 1635-1642.

8. Herrmann BW, Forsen JW. Simultaneous intracranial and orbital complications of acute rhinosinusitis in children. Int J Pediatr Otorhinolaryngol 2004; 68: 619-625.

9. Ferguson MP, McNab AA. Current treatment and outcome in orbital cellulitis. Aust N Z J Ophthalmol 1999; 27: 375-379.

10. Nwaorgu OGB, Awobem JF, Onakoya PA, Awobem AA. Orbital cellulitis complicating sinusitis: a 15-year review. Nigerian J Surg Res 2004; 6: 14-16.

11. Tshifularo M, Monama GM. Complications of inflammatory sinusitis in children: institutional review. SA Fam Pract 2006; 48: 16.

12. Singh B, Van Dellen J, Ramjettan S, Maharaj TJ. Sinogenic intracranial complications. J Laryngol Otol. 1995; 109: 945-950.

13. Chaudhry IA, Shamsi FA, Elzaridi E, Al-Rashed W, Al-Amri A, Al-Anezi F, Arat YO, Holck DE.Outcome of treated orbital cellulitis in a tertiary eye care center in the middle East. Ophthalmology 2007; $114: 345-354$ 
14. Eviatar E, Gavriel H, Pitaro K, Vaiman M, Goldman M, Kessler A. Conservative treatment in rhinosinusitis orbital complications in children aged 2 years and younger. Rhinology. 2008; 46: 334-337.

15. Eufinger H, Machtens E. Purulent pansinusitis, orbital cellulitis and rhinogenic intracranial complications. J Craniomaxillofac Surg 2001; 29: 111-117.

16. Reynolds DJ, Kodsi SR, Rubin SE, Rodgers IR. Intracranial infection associated with preseptal and orbital cellulitis in the pediatric patient. J AAPOS 2003; 7: 413-417.

17. Brijlal Meena MK, Sharma P. Epidural empyema secondary to orbital cellulitis: a case report. Eur J Ophthalmol 2007; 17: 841-843.

18. Jones NS, Walker JL, Bassi S, Jones T, Punt J. The intracranial complications of rhinosinusitis: can they be prevented? Laryngoscope 2002; 112: 59-63.

19. Howe L, Jones NS. Guidelines for the management of periorbital cellulitis/abscess. Clin Otolaryngol Allied Sci 2004; 29: 725-728.

20. Gosepath J, Brieger J, Vlachtsis K, Mann WJ. Fungal DNA is present in tissue specimens of patients with chronic rhinosinusitis. Am J Rhinol 2004; 18: 9-13.
21. Yen MT, Yen KG. Effect of corticosteroids in the acute management of pediatric orbital cellulitis with subperiosteal abscess. Ophthal Plast Reconstr Surg 2005; 21: 363-367.

Jan Kastner

Dept. of Otorhinolaryngology and Head and Neck Surgery University Hospital Motol, 1st Medical Faculty

Charles University

V Uvalu 84

15006 Prague

Czech Republic

Tel: $+42-60-4268166$

Fax: +42-22-443 4319

E-mail: jkastner@email.cz,jkastner@uni-mainz.de 